\title{
Erratum to: Automation of Finite Element Methods
}

Jože Korelc and Peter Wriggers

\section{Erratum to:}

J. Korelc and P. Wriggers, Automation of Finite Element Methods, DOI 10.1007/978-3-319-39005-5

An error in the production process unfortunately led to publication of this book prematurely, before incorporation of the final corrections. The version supplied here has been corrected and approved by the authors.

The updated original online version for this book can be found at DOI 10.1007/978-3-319-39005-5

\footnotetext{
J. Korelc $(\bowtie)$

Faculty of Civil and Geodetic Engineering, University of Ljubljana, Ljubljana, Slovenia

e-mail: jkorelc@fgg.uni-lj.si

P. Wriggers

Leibniz University Hannover, Hannover, Germany

e-mail: wriggers@ikm.uni-hannover.de 